\section{Commentary: Indocyanine green: The Green Lantern of congenital heart surgery?}

\author{
Sitaram M. Emani, MD
}

Angiography and computed tomography have been utilized to assess patency and location of vascular structures for many years. A surgeon must interpret the results of these studies and superimpose the images onto the surgical field with a healthy imagination or a hybrid operating room suite. The unique fluorescent property of indocyanine green (ICG) has been leveraged in imaging technology to allow intraoperative visualization of various vascular structures and assist with surgical planning and assessment of technical results. Said and colleagues ${ }^{1}$ demonstrate their experience with the technology in myriad clinical situations. There are several important lessons to be gleaned.

Under what clinical circumstances is this methodology most useful? In its current form, the information gathered is qualitative rather than quantitative, so imaging can identify gross features such as location of a coronary artery or presence of perfusion in a coronary artery bed. We have used the system most commonly to identify the location of epicardial coronary arteries and determine the site of ventriculotomy in reoperative cardiac surgery when locations of key coronary arteries are not obvious. ${ }^{2}$ It may be useful in identifying the location of coronary vasculature within a cardiac tumor to avoid injury during debridement. The depth of penetration is relatively shallow, thus coronary arteries that are embedded within myocardium, sheets of scar tissue, or tumor may not light up. The system can also demonstrate if there is perfusion to a particular region by revealing blush of ICG fluorescence in the distal perfusing

From the Department of Cardiovascular Surgery, Boston Children's Hospital, Boston, Mass.

Disclosures: Dr Emani is a consultant for Cheisi Pharmaeuticals.

The Journal policy requires editors and reviewers to disclose conflicts of interest and to decline handling or reviewing manuscripts for which they may have a conflict of interest. The editors and reviewers of this article have no conflicts of interest.

Received for publication March 14, 2021; revisions received May 14, 2021; accepted for publication May 19, 2021; available ahead of print May 23, 2021.

Address for reprints: Sitaram M. Emani, MD, Department of Cardiovascular Surgery, Boston Children's Hospital, 300 Longwood Ave, Boston, MA 02115 (E-mail:

Sitaram.emani@cardio.chboston.org).

JTCVS Techniques 2021;8:158-9

2666-2507

Copyright (C) 2021 The Author(s). Published by Elsevier Inc. on behalf of The American Association for Thoracic Surgery. This is an open access article under the CC BY-NC-ND license (http://creativecommons.org/licenses/by-nc-nd/4.0/).

https://doi.org/10.1016/j.xjtc.2021.05.018

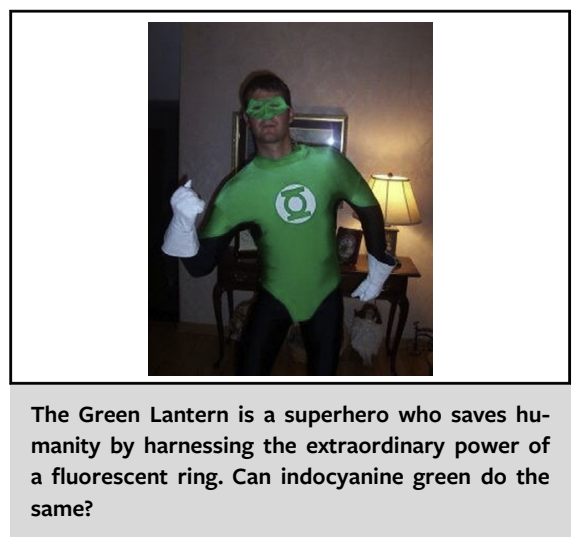

CENTRAL MESSAGE

Intraoperative visualization of

coronary arteries with fluores-

cent indocyanine green has po-

tential to guide surgical

management. The protocols for

its use are being optimized.

myocardium. It may thus be useful in excluding complete occlusion of a particular vessel.

The potential of this technology to provide detail regarding adequacy of an anastomosis, or stenosis in a vessel (beyond complete occlusion) remains uncertain. The technique is extremely sensitive to the quantity of contrast administered as well as the speed at which it is administered. Fluorescence can scatter and impair resolution, making a relatively narrow anastomosis look wide open. Even in the face of a significant stenosis, one can demonstrate distal perfusion within the vascular bed. As the technology matures and the technique is standardized, this will likely improve. Although the authors utilized this technique to assess Blalock-Taussig shunt patency (with venous grafts), ICG cannot penetrate polytetrafluoroethylene or polyethylene terephthalate, thus limiting its use in this clinical scenario.

Said and colleagues ${ }^{1}$ provide important details regarding dilution of ICG, dose, and method of delivery, The ICG can be administered into the aortic root in a crossclamped or perfusing heart after institution of cardiopulmonary bypass. The presence of aortic insufficiency may affect the dosing and timing of administration. As mentioned by the authors, the variables that can be modified include quantity of ICG administered, the rate at which it is administered, and its concentration. To adequately visualize the structure, several 
injections of varying injection rates and volumes may be necessary to obtain optimal visualization of the target structures. Short small bursts will delineate anatomy with high resolution for a brief period of time, whereas long slow infusions will provide prolonged visualization with less distinct borders. The contrast can be washed out by administration of additional cardioplegia in a crossclamped heart, or by allowing washout in the perfused heart, and imaging can be repeated. Fortunately, ICG has a favorable safety profile, and even multiple injections can be performed without reaching the upper limit of dosing.
One can certainly imagine the possible applications of intraoperative imaging with ICG. We look forward to welldesigned prospective clinical trials that standardize the technique and demonstrate its advantages for our vulnerable patients.

\section{References}

1. Said SM, Marey G, Hiremath G. Intraoperative fluorescence with indocyanine green in congenital cardiac surgery: potential applications of a novel technology. J Thorac Cardiovasc Surg Tech. 2021:8:144-55.

2. Feins EN, Si MS, Baird CW, Emani SM. Intraoperative coronary artery imaging for planning. Semin Thorac Cardiovasc Surg. 2020;23:11-6. 